\title{
A Respiration Sensor for a Chest-Strap Based Wireless Body Sensor
}

\author{
Marc Hesse, Peter Christ, Timm Hörmann, Ulrich Rückert \\ Cognitronics and Sensor Systems Group \\ CITEC, Bielefeld University, 33619 Bielefeld \\ Email: \{mhesse, pchrist, thoermann, rueckert\}@cit-ec.uni-bielefeld.de
}

\begin{abstract}
In this paper we present a respiration sensor suitable for an integration into a wireless body sensor worn around the chest. The thorax expansion and contraction during in- and exhalation is captured using a force-sensing resistor. Based on the captured thoracic movements, the breaths are determined with a peak detection algorithm. For evaluation, a treadmill experiment with five subjects was conducted using an ergospirometry system as a reference. Overall, an average deviation of $\mathbf{- 0 . 3 2} \pm 0.68 \mathrm{~min}^{-1}$ in the respiration rate between the ergospirometry and our sensor was observed. In general, the captured thoracic movements showed breaths as distinctive oscillations, but in some cases a non-optimal pressure transfer between thorax and sensor was observed. Therefore, a mechanical housing mechanism was developed. A comparison of our construction with a respiratory inductance plethysmography (RIP)based sensor shows a close relationship with the captured thoracic movements during normal and deep respiration.
\end{abstract}

\section{INTRODUCTION}

We are developing a wireless body sensor (BG-V4.2) for applications in sports, preventive medicine and daily life. Our body sensor is worn around the chest using a strap, which is already a widely established, easily manageable and reliable method for heart-rate monitoring. Currently, our body sensor allows measuring a person's ECG, skin temperature and body accelerations (see Fig. 1). We plan to additionally capture respiratory information such as the respiration rate, which is an important parameter within sports-exercise and preventive stress management applications. Our system is suitable to record a person's thoracic movements and to derive the respiration rate. It is applicable in natural environments ranging from sedentary to sports activities.

There are sensors available to measure the oral or nasal respiratory airflow using different detection modalities: pressure fluctuations, hot wire anemometry, passive temperature sensing, humidity sensor or carbon dioxide detectors [1]. Other approaches try to measure the respiration with indirect methods. Although the physiological respiration is a complex process, it can be approximated with two degrees of freedom: volume change in rib cage and abdomen [2]. The resulting chest and abdominal motion can be captured using respiratory inductance plethysmography (RIP), transthoracic impedance plethysmography, a magnetometer, a strain gauge or a pressure sensor [1]. Additionally, the respiration rate can be derived from the analysis of electrocardiogram or photoplethysmogram waveforms [3]. The indirect methods capturing the

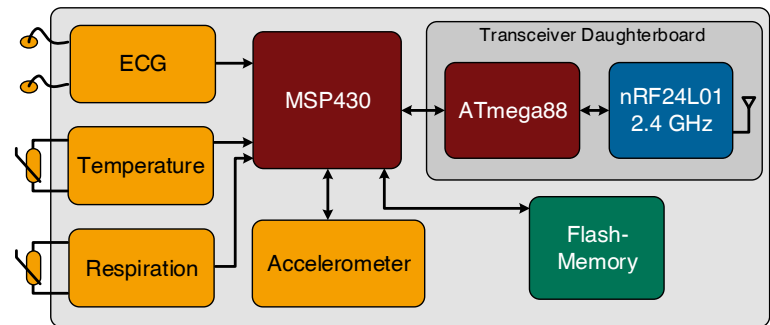

Fig. 1: Technical block diagram of our wireless body sensor BG-V4.2.

thoracic and abdominal movements are increasingly used within mobile systems, which are capable of measuring the respiration rate, for example, two mobile systems are developed for biofeedback applications. The first one uses textilebased sensors consisting of a piezoresistive material [4]. A shirt with a number of these sensors has been developed to measure thoracic and abdominal expansions. The second system consists of a waistband with an embedded stretch sensor held in place by two clips [5].

This paper is organized as follows: Section II introduces the current prototype of our wireless body sensor in which the respiration sensor is integrated. Its technical realization is described in Section III. An overview of the conducted experiment for data collection is given in Section IV. Furthermore, the recorded thoracic movements are analyzed and an optimized version of the mechanical housing mechanism of the respiration sensor is described. Section V explains the algorithm for determining the respiration rate based on the thoracic movements. In Section VI the results of our evaluation on the data of the experiment are given and compared to reference values of an ergospirometry system. In Section VII a summary and a prospect on our future work is given.

\section{TECHNICAL BACKGROUND}

Our current prototype of the wireless body sensor (BGV4.2) allows to record a person's ECG, skin temperature and body accelerations (see Fig. 1). The accelerometers integrates three sensitive axes and has an adjustable measurement range ( \pm 6 to $\pm 24 \mathrm{~g}$ ) in order to capture accelerations of daily activities as well as sports. An MSP430FG4618 microcontroller from Texas Instruments is utilized to execute the 
firmware and to perform signal processing tasks such as an estimation of the person's walking and running velocity [6], [7]. Data is stored in a flash memory and is optionally transmitted through a wireless interface (nRF24L01) to a base station [8]. The whole system is embedded into a single module which can be used with a conventional chest strap and is powered by a single CR2025 coin cell.

\section{CONSTRUCTION OF RESPIRATION SENSOR}

Airflow detecting respiration sensors require more patient co-operation and may limit mobility [1]; in particular they can affect breathing patterns by decreasing the respiration rate and increasing the tidal volume [9]. However, for an integration into a mobile body sensor a non-clinical, robust and multiuse functionality is an important issue [10]. Chest straps are already widely accepted within heart-rate monitoring and they are suitable to be worn during daily activities as well as sports. Therefore, we integrated our sensor into a chest strap which can be easily attached by the user. For the realization of the respiration sensor we decided against RIP-based sensors as they are still relatively expensive and difficult to handle [1]. Furthermore, we excluded impedance plethysmography because of its sensitivity to body movements [11] which would result in motion related artifacts during physical exercise. Our initial choice was a piezoelectric film, but during preliminary tests we observed a high edge steepness in relation to the applied pressure during in- and exhalation. This problem was not found with a force-sensing resistor which reflected the thoracic movements very well and proved to be a robust solution. We chose the FlexiForce A201 from Tekscan for our respiration sensor.

Using an operational amplifier with a subsequent low-pass filter $(\mathrm{fc}=12.54 \mathrm{~Hz})$, the force-sensing resistor is connected to an ADC-channel of the body sensor's microcontroller. An additional digital low-pass filter with $\mathrm{fc}=0.6 \mathrm{~Hz}$ is applied to remove signal noise and artefacts. The chosen cut-off frequency is suitable for slow as well as fast respiration during daily activities and exercise of light to medium intensity. For its integration into the chest-strap, the force-sensing resistor is integrated in a simple mechanical housing mechanism, which basically consists of two quadratic plates $(1,5 \mathrm{~cm}$ length), to avoid being bent or pushed into the soft strap.

\section{EXPERIMENT: TREADMILL EXERCISE}

\section{A. Data Collection}

For the evaluation of the respiration sensor, data was collected in a treadmill experiment with five subjects (one female) who completed the following protocol consisting of eight stages where each stage continued for $3 \mathrm{~min}$ : 0 - 3 5-7-9-11-5-0 km/h. During the experiment, the respiration sensor was placed on the skin under the chest-strap located between the linea medioclavicularis and the linea axillaris anterior. The signals of the sensor were sampled with $100 \mathrm{~Hz}$ and saved in the flash-memory of the body sensor. For the acquisition of reference values, the respiration rate
TABLE I: Average contact pressure and its variations due to the thoracic movements resulting from in- and exhalation.

\begin{tabular}{ccc}
\hline Subject & mean [lsb] & std [lsb] \\
\hline S1 & 2037 & 448 \\
S2 & 2473 & 768 \\
S3 & 2221 & 569 \\
S4 & 2196 & 199 \\
S5 & 1861 & 68 \\
\hline
\end{tabular}

was additionally recorded $(0.1 \mathrm{~Hz}$ sampling rate $)$ using an ergospirometry system (CORTEX Metalyzer).

\section{B. Analysis of Thoracic Movements}

The signals of the five subjects showed rhythmic oscillations corresponding to the in- and exhalations. However, we observed differences in the signals' amplitudes between the subjects. In particular, the breaths of subjects S4 and S5 were not as distinctively visible as of the other subjects. We determined the contact pressure of the respiration sensor and the signal's variations by calculating the mean and the standard deviation of the force-sensing resistor's output for each subject (see Tab. I). The calculated mean corresponds to the estimated contact pressure, which is based on the ratio between the measured lengths of the chest straps and the chest girths. In comparison to the other subjects, the contact pressure was only lower for S5. Therefore, the observed amplitude difference can not be explained by an insufficient contact pressure. However, the signals of S4 and S5 showed a significantly reduced standard deviation, which correlates to a lower signal amplitude. An explanation could be, that the simple mechanical housing mechanism was insufficient, so that the sensitive surface of the sensor was pushed into the intercostal space. To assure a correct placement and a robust pressure transfer, we optimized the housing mechanism.

\section{Optimization of Mechanical Housing Mechanism}

In order to improve the measurement of the thoracic movements, the characteristics of the chosen force-sensing resistor were further evaluated. An experimental setup was arranged, in which it was possible to apply precise forces to the sensor. A total of nine sensors were tested. The tests included variations of the applied force $(0-4.6 \mathrm{~N})$ and the use of different geometrical shapes to apply these forces. Best results in terms of linearity were achieved with a round shape, which covered nearly the whole sensitive surface of the sensor. Taking this into account, an optimized mechanical housing mechanism was constructed and 3D-printed. It consists of three parts. The bottom and middle parts enclose the force-sensing resistor and are stuck and glued together. The top part features a round shape which directs the thoracic movements to the sensitive sensor surface. The top part is loosely coupled with the middle part. The optimized mechanical housing mechanism with the integrated force-sensing resistor (see Fig. 2) is fixed on the chest strap, so that it is located on the right chest side. 


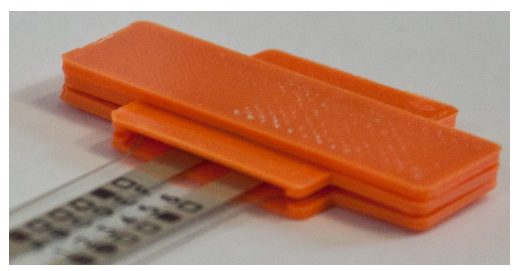

Fig. 2: Picture of the optimized mechanical housing mechanism $(30 \mathrm{~mm} \times 19.5 \mathrm{~mm} \times 2.8 \mathrm{~mm})$ with the integrated forcesensing resistor. The body sensor is worn on a chest strap with the respiration sensor located on the right chest side.
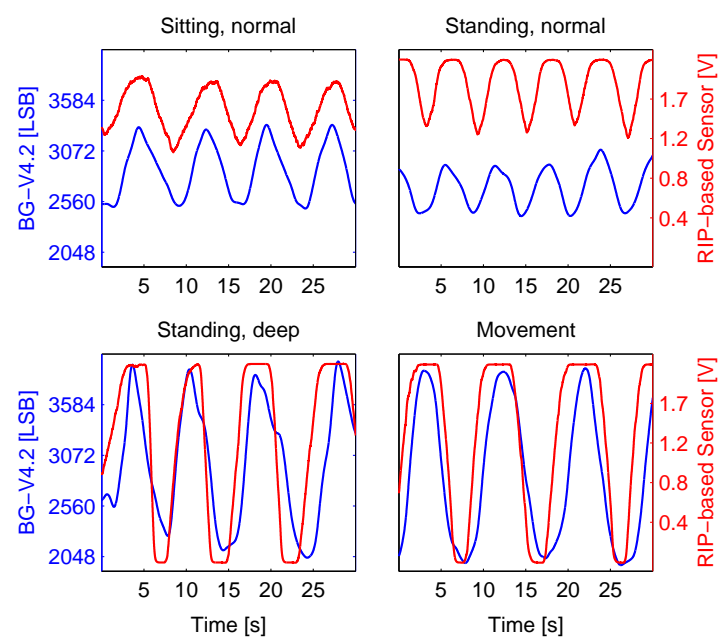

Fig. 3: Comparison of thoracic movements recorded with our respiration sensor enclosed in the optimized mechanical housing mechanism (BG-V4.2) and the RIP-based respiration sensor.

We validated the functionality of our construction in a comparison with an RIP-based respiration sensor which is part of the QuickAmp system from Brain Products. The validation covered measurements while standing and moving. Additionally, normal and deep respiration were considered. The comparison between the RIP-based sensor and our construction showed a close agreement regarding the captured thoracic movements (see Fig. 3). Our solution does not only improve the pressure transfer between thorax and sensor, but also facilitates the assembly of the respiration sensor and therefore enhances the fail-safety and the robustness of the measurement.

The following results in this paper are however based on the original setup (see Sec. III) as the optimized version was developed after the data collection.

\section{Determination of RESPiRATION RATE}

Initially, we evaluated the peakDet-algorithm of E. Billauer [12] to segment the captured thoracic movements into single breaths. However, we found the constant amplitude threshold of the peak-detection to be disadvantageous because of the variability in the signal's amplitude and frequency,

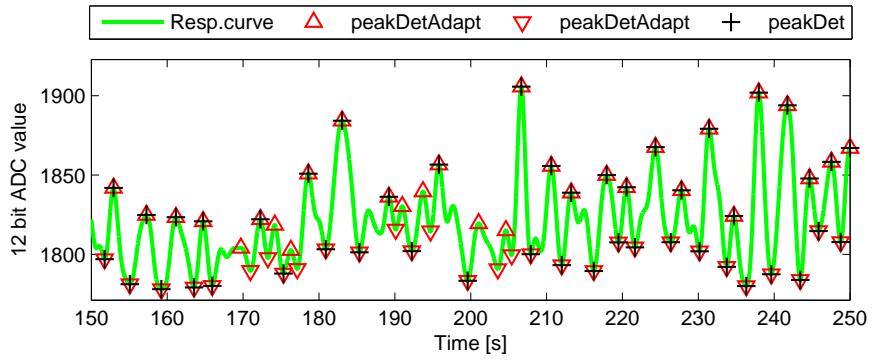

(a) Low-pass filtered ( $\mathrm{fc}=0.6 \mathrm{~Hz}) \mathrm{ADC}$ value with markers of the two peak-detection methods.

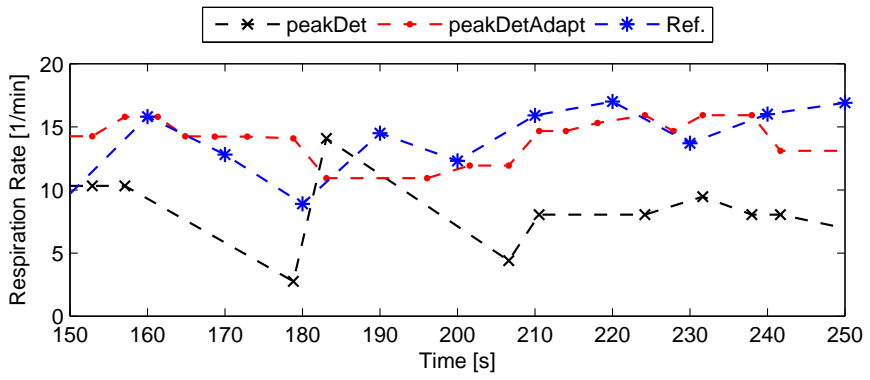

(b) Comparison of the derived respiration rate based on the two peakdetection methods with the reference values from the ergospirometry.

Fig. 4: Output of the two peak-detection methods on the recorded thoracic movements of subject S5 (a) and the determined respiration rates (b). The results based on the peakDetAdapt provide a closer agreement with the ergospirometry reference.

caused by normal and deep as well as slow and fast respiration. In order to overcome this drawback, we extended the algorithm to adapt its detection of peaks dynamically to different signal amplitudes and frequencies (peakDetAdapt). The peak detection is carried out within time windows of collected sensor data. The length of the time window is altered accordingly to the interval between two successively detected peaks. Simultaneously the amplitude threshold is updated every time a new peak is detected or the time window has elapsed. Each new threshold results from the weighted sum of the old threshold (weight $=0.9$ ) and the newly observed peak-to-peak value (weight $=0.1$ ). The threshold's minimum is bounded by the noise of the respiration sensor's measurement chain $(75 \mathrm{LSB})$. Figure $4 \mathrm{a}$ shows data from subject S5 as an example with the detected peaks of both algorithms. The comparison of the two algorithms showed a closer agreement for respiration rates determined using our peakDetAdapt with the outcome of the ergospirometry system (see Fig. 4b).

\section{RESUlTS}

In order to compare our sensor to the ergospirometry system used as a reference in the treadmill experiment, the proposed peakDetAdapt algorithm was applied to calculate the respiration rate based on the captured thoracic movements. The following results were obtained by subtracting the ergospirometry values from our determined respiration rate. Overall, 


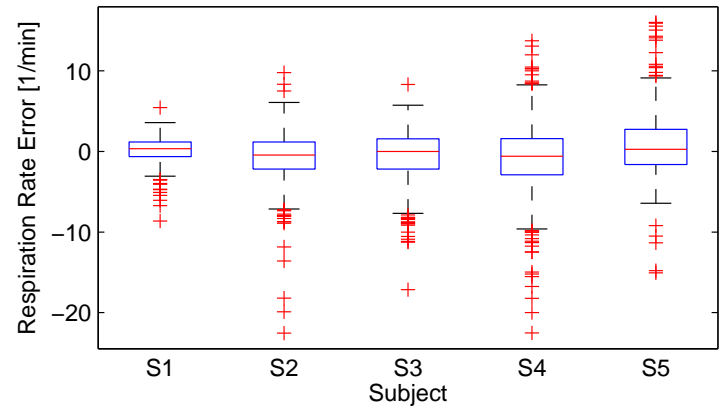

Fig. 5: Box-and-whisker plots of differences between respiration rates determined with our respiration sensor (peakDetAdapt) of the BG-V4.2 and the ergospirometry system.

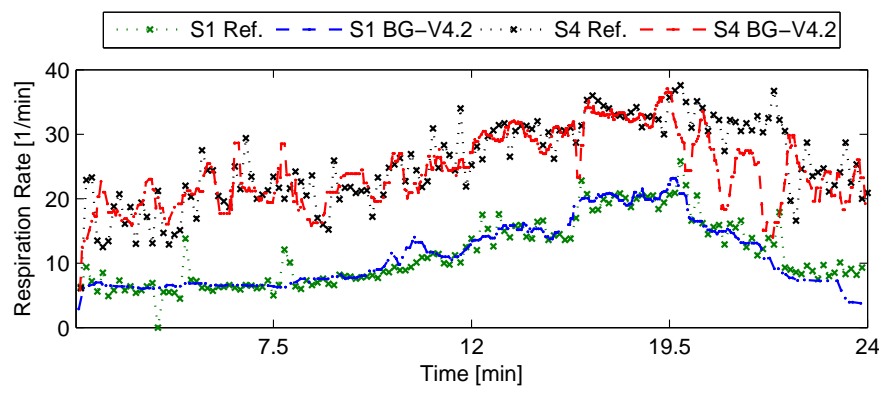

Fig. 6: Comparison of respiration rate curves determined with our body sensor (BG-V4.2) and the ergopirometry system as a reference. Shown are the results for subjects S1 and S4.

we observed an average deviation of $-0.32 \pm 0.68 \mathrm{~min}^{-1}$. The results are summarized as box-and-whisker plots (see Fig. 5).

While the low average deviation represents a satisfying overall outcome, there are some stages in the experiment where the deviation between our system and the ergospirometry is quite large. Most outliers were observed with subjects S4 and S5. An example can be seen in Figure 6, where the calculated respiration rates of subjects $\mathrm{S} 1$ and $\mathrm{S} 4$ are plotted along with the ergospirometry values. The calculated respiration rates for subject S1 show a close agreement with the ergospirometry reference (min: -8.85 , max: 5.44, std: 1.74 ), but the respiration rate for subject S4 exhibits major differences (min: -22.52, max: 13.74, std: 4.47) with significant variations between minutes 19 and 23. These variations are, as discussed earlier, caused by an insufficient pressure transfer between thorax and sensor. To assure a robust recording of the respiration rate, the optimized mechanical housing mechanism was developed.

\section{Summary AND FUtURE WORK}

We presented a chest-strap based respiration sensor consisting of a force-sensing resistor integrated into a mechanical housing mechanism for transferring the thoracic movements onto the sensitive area. The functionality of the housing mechanism was tested by comparing our sensor to a stationary respiratory inductance plethysmography (RIP)-based sensor. The respiration sensor was integrated into the chest strap of our body sensor allowing to record in- and exhalation movements without disturbances during daily activities or sports. An amplitude and frequency adaptive peak-detection algorithm was realised to determine the respiration rate. In a treadmill experiment with five subjects and velocities of up to $11 \mathrm{~km} / \mathrm{h}$ we observed an average deviation of the respiration of $-0.32 \pm 0.68 \mathrm{~min}^{-1}$ in comparison to an ergospirometry system as a reference. Our respiration sensor can be integrated into other body sensors as well; it requires a low-pass filter circuit with a subsequent operational amplifier and an ADC-port.

Currently we are implementing our peak detection algorithm (peakDetAdapt) on the microcontroller, so that we are able to determine the respiration rate in real-time. In future work we plan to further validate our sensor by a repeated treadmill experiment with a higher number of subjects. Afterwards field studies are planned in order to evaluate the performance of our sensor during daily life activities.

\section{ACKNOWLEDGMENT}

This research was supported by the DFG CoE 277: Cognitive Interaction Technology (CITEC) and the German Federal Ministry of Education and Research (BMBF) within the Leading-Edge Cluster 'Intelligent Technical Systems OstWestfalenLippe" (it's OWL), managed by the Project Management Agency Karlsruhe (PTKA), and the BMBF funded project ALUBAR. The authors are responsible for the contents of this publication. The authors would like to thank the Department of Sport Science at Bielefeld University for the use of their ergospirometry lab.

\section{REFERENCES}

[1] P. Oberg, et al., Sensors Applications, Sensors in Medicine and Health Care, ser. Sensors Applications. Wiley, 2006.

[2] K. Konno and J. Mead, "Measurement of the separate volume changes of rib cage and abdomen during breathing," Journal of Applied Physiology, vol. 22 , no. 3, pp. 407-22, 1967.

[3] D. Meredith, et al., "Photoplethysmographic derivation of respiratory rate: a review of relevant physiology," Journal of Medical Engineering and Technology, vol. 36, no. 1, pp. 1-7, 2012.

[4] E. Mitchell, et al., "Breathing feedback system with wearable textile sensors," in Int. Conf. on Body Sensor Networks, Jun. 2010, pp. 56-61.

[5] N. Moraveji, et al., "Peripheral paced respiration: influencing user physiology during information work," in Proc. of the 24th annual ACM symposium on User interface software and technology. ACM, 2011, pp. $423-428$.

[6] P. Christ, et al., "An approach for determining linear velocities of athletes from acceleration measurements using a neural network," in Proc. of the 6th IASTED Int. Conf. on Biomechanics, B. M. M. Hamza, Ed. ACTA Press, Nov. 2011, pp. 105-112.

[7] P. Christ, et al., "Pareto-optimal signal processing on low-power microprocessors," in Proc. of the 12th IEEE Int. Conf. on SENSORS, Nov. 2013, pp. 1843-1846.

[8] P. Christ, et al., "Performance analysis of the nRF24L01 ultra-low-power transceiver in a multi-transmitter and multi-receiver scenario," in Proc. of the 10th IEEE Int. Conf. on SENSORS. IEEE, Oct. 2011, pp. 12051208.

[9] R. Gilbert, et al., "Changes in tidal volume, frequency, and ventilation induced by their measurement." Journal of Applied Physiology, vol. 33, no. 2, pp. 252-254, 1972.

[10] A. Pantelopoulos and N. Bourbakis, "A survey on wearable sensor-based systems for health monitoring and prognosis," IEEE Trans. on Systems, Man, and Cybernetics, Part C: Applications and Reviews, vol. 40, no. 1, pp. 1-12, Jan. 2010.

[11] J. Rosell, et al., "Reduction of motion artifacts using a two-frequency impedance plethysmograph and adaptive filtering," IEEE Trans. on Biomedical Engineering, vol. 42, no. 10, pp. 1044-1048, 1995. 
[12] E. Billauer. (2012) peakdet: Peak detection using matlab (v.3.4.05). [Online]. Available: http://billauer.co.il/peakdet.html 